\title{
Laxative use and incident falls, fractures and change in bone mineral density in postmenopausal women: results from the Women's Health Initiative
}

\author{
Bernhard Haring ${ }^{1 *}$, Mary Pettinger ${ }^{2}$, Jennifer W Bea ${ }^{3}$, Jean Wactawski-Wende ${ }^{4}$, Ryan M Carnahan ${ }^{5}$,
} Judith K Ockene ${ }^{6}$, Moritz Wyler von Ballmoos ${ }^{7}$, Robert B Wallace ${ }^{5}$ and Sylvia Wassertheil-Smoller ${ }^{8}$

\begin{abstract}
Background: Laxatives are among the most widely used over-the-counter medications in the United States but studies examining their potential hazardous side effects are sparse. Associations between laxative use and risk for fractures and change in bone mineral density [BMD] have not previously been investigated.

Methods: This prospective analysis included 161,808 postmenopausal women (8907 users and 151,497 nonusers of laxatives) enrolled in the WHI Observational Study and Clinical Trials. Women were recruited from October 1, 1993, to December 31, 1998, at 40 clinical centers in the United States and were eligible if they were 50 to 79 years old and were postmenopausal at the time of enrollment. Medication inventories were obtained during in-person interviews at baseline and at the 3-year follow-up visit on everyone. Data on self-reported falls ( $\geq 2$ ), fractures (hip and total fractures) were used. BMD was determined at baseline and year 3 at 3 of the 40 clinical centers of the WHI.

Results: Age-adjusted rates of hip fractures and total fractures, but not for falls were similar between laxative users and non-users regardless of duration of laxative use. The multivariate-adjusted hazard ratios for any laxative use were 1.06 (95\% confidence interval [Cl], 1.03-1.10) for falls, 1.02 (95\% Cl, 0.85-1.22) for hip fractures and 1.01 (95\% Cl, 0.96-1.07) for total fractures. The BMD levels did not statistically differ between laxative users and nonusers at any skeletal site after 3-years intake.

Conclusion: These findings support a modest association between laxative use and increase in the risk of falls but not for fractures. Its use did not decrease bone mineral density levels in postmenopausal women. Maintaining physical functioning, and providing adequate treatment of comorbidities that predispose individuals for falls should be considered as first measures to avoid potential negative consequences associated with laxative use.
\end{abstract}

Keywords: Laxative use, Falls, Fractures, Bone mineral density, Aging

\section{Background}

Laxatives belong to one of the most frequently sold overthe-counter pharmacy sales categories in the United States [1]. In 2011, consumers spent approximately $\$ 875$ million on laxative drug products [1]. This easy availability can facilitate use without indication, long-term use, inappropriate dosing and even abuse of these medicines [2-4]. Electrolyte

\footnotetext{
*Correspondence: Haring_B@medizin.uni-wuerzburg.de

'Department of Internal Medicine I, Comprehensive Heart Failure Center, University of Würzburg, Oberdürrbacher Strasse 6, 97080, Würzburg, Germany Full list of author information is available at the end of the article
}

and fluid imbalances have been reported as potential negative consequences and side-effects of laxative use [2-7]. These medical conditions are known causes of muscle weakness, lassitude, cardiac arrhythmias, and osteoporosis that may increase the risk for falls and fractures [8-14]. In fact, use of a wide range of prescription drugs such as loop diuretics or antiepileptics have already been shown to be associated with falls, fractures and even changes in bone mineral density as these drugs may alter fluid and electrolyte metabolism (e.g. hyponatremia, hypocalemia) and even vitamin D absorption [10,15-19].

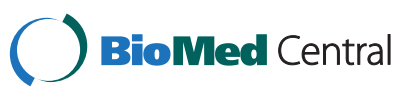


To this point, prior epidemiological studies suggest that laxative use increases the risk for falls [2,20-29]. However, these studies were limited by small numbers of falls, lack of data on important potential confounders and short follow-up period [20,22,27,29]. Additionally, although electrolyte and fluid imbalances, vitamin malabsorption as well as a history of falls are important risk factors for changes in bone mineral density and fractures $[9,10,30-39]$, the effect of laxative use on these bone outcomes remains to be determined.

Based on current literature laxative users may be characterized by several different groups $[4,40,41]$. The first and by far largest group is made up of individuals who are generally middle aged or older who begin using laxatives when constipated. The second group consists of individuals suffering from an eating disorder such as anorexia or bulimia nervosa. Third, individuals engaged in certain types of sports training may be inclined to be users. The fourth group includes surreptitious laxative abusers who use the drugs to cause factitious diarrhea. As constipation occurs more frequently in the female population and it becomes more prevalent with increasing age [42-45], the role of laxative use in postmenopausal women is of particular interest.

Our aim is to evaluate a possible association of laxative intake with the incidence of falls, total fractures, hip fractures and change in bone mineral density in postmenopausal women. Such information may provide insight into the safety profile and potential hazards of a widely used medication class and thereby be of high public health relevance.

\section{Methods}

\section{Study population}

The study population consisted of 161,808 women enrolled in the Women's Health Initiative (WHI) Observational Study (OS) $(n=93,676)$ and Clinical Trials (CTs) $(n=68,132)$. Details of the study population have been described elsewhere [46-50]. For this analysis, 160,404 women with no prior hip fracture (OS: $n=92,789 / C T$ : $\mathrm{n}=67,615$ ) were included. Women were recruited from October 1, 1993, to December 31, 1998, at 40 clinical centers in the United States and were eligible if they were 50 to 79 years old and were postmenopausal at the time of enrollment. In addition to age and menopausal status, eligibility criteria for the clinical trial $(\mathrm{CT})$ and observational study (OS) included ability and willingness to provide written informed consent and an agreement to reside in the area for at least 3 years after enrollment [51], [46]. Participants were subsequently followed up through March 31, 2005, for an average of 7.8 years (OS: 7.6 years /CT: 8 years). Among 160,404 women, 7421 (4.6\%) were lost to follow-up (OS: $n=4451 / C T: n=2970)$, whereas 9709 (6.1\%) deceased (OS: $n=6146 / C T: n=3563)$.
OS participants were scheduled for two clinic visits at baseline and year 3 of follow-up compared to CT participants who were scheduled for clinic visits at years 1, 3, 6 and 9. To update selected exposures and ascertain medical outcomes participants in the OS were mailed annual questionnaires whereas women in the CTs were sent semiannual forms to ensure the timely update of medical histories [46]. All protocols were approved by institutional review boards at participating institutions.

\section{Exposure assessment}

Current medication use was assessed by asking the participants to present all containers for medications taken for 2 weeks prior to clinic visit [46]. Clinic interviewers then entered each medication into the WHI database, which assigned drug codes using Medispan software (First DataBank, Inc., San Bruno, California). For this analysis, we used baseline information on current laxative use including label product name, label generic name, dosage form, strength and duration (WHI Form 44: Current Medications). Duration of use was examined in three categories ( $<1$ year, 1 to 3 years, or $>3$ years). Since the medication class of laxatives is a heterogeneous entity with various different kinds of pharmacologically active substances and with many commercially available laxative products consisting of two or more active pharmacological compounds, we investigated the effect of 'any (compound containing) laxative' on our outcomes of interest. We additionally stratified laxatives by the underlying containing substances into five categories ['oral stimulant containing,' 'fiber containing, 'osmotic containing,' 'stool softener containing' and 'lubricant containing' laxative] to potentially gain information on the distribution of these categories being used and the effects on our outcomes of interest. However, as we are lacking information on the exact composition and pharmacological impact of each compound of the various commercially available mixture products, for analyses these categories were not mutually exclusive.

\section{Outcome assessment and other covariates}

As primary outcome measures, we assessed the incidence of falls and fractures (total and hip fractures) and change in bone mineral density. Incident falls and fractures were ascertained prospectively using annual (OS) or semiannual (CT) self-report questionnaires. Fall history was obtained by asking about the number of times the participant fell or landed on the ground (excluding falls owing to sports) in the interval since the last medical history update (WHI Form 33: Medical History). A participant was identified as having a history of falls if she reported 2 or more falls per year as previously defined in epidemiologic studies $[15,16,52,53]$. In WHI CTs, all fracture outcomes were verified by central review of 
radiology reports whereas in the WHI OS, only hip fractures were centrally adjudicated for all women.

Bone mineral density (BMD) at the total hip, posterioranterior spine, and total body was measured at baseline at 3 of the 40 clinical centers of the WHI (Pittsburgh, Pennsylvania; Birmingham, Alabama; and Phoenix and Tucson, Arizona) among 10,833 women with dual-energy x-ray absorptiometry using a Hologic QDR densitometer (Hologic Inc, Waltham, Massachusetts). We determined change in BMD by laxative use at these sites from baseline to year 3. Information on all covariates was assessed via self-report, clinic interview or by physical measure at baseline [54-56].

\section{Statistical analysis}

The characteristics of women using laxatives at baseline were compared with nonusers [Table 1]. Differences between laxative users and nonusers were compared using $X^{2}$ statistics for categorical variables and $t$-test or ANOVA, as appropriate, for continuous variables. Clinical outcomes were summarized according to laxative use and its various subgroups [Table 2]. Age-adjusted incidence rates of falls, total and hip fractures per 1000 person-years were calculated according to duration of laxative use by direct-adjustment based on the 5-year age distribution of the total CTs+OS sample $(n=161,808)$ [Additional file 1: Table S1]. Hazard ratios (HRs) for the risk of falls $(\geq 2)$ and total or hip fractures associated with laxative use at baseline as compared with no laxative use were obtained by adjusted Cox proportional hazards analyses using time from enrollment to the first occurrence of the event as 'time' in the models [Table 3]. Two models were formed for each outcome to examine the effect of potential confounding $[15,16,57,58]$. Model 1 was stratified by 10 -year age intervals and OS vs. CT cohorts, and adjusted for linear age, race/ethnicity, and randomization assignment for WHI-CTs. Model 2A additionally adjusted for BMI, smoking, physical activity, self-reported health, treated diabetes mellitus, history of fracture at age $\geq 55$ years, corticosteroid use, physical function score, number of chronic medical conditions (including treated diabetes mellitus, stroke, any cancer, CVD, arthritis, hypertension, and emphysema), number of psychoactive medications (including anxiolytics, hypnotics, antidepressants, antipsychotics, and antiepileptic agents), use of HT and bisphosphonates. Model 2B also included history of falls $(\geq 2)$. Similarly, we constructed adjusted hazards ratio of falls and bone outcomes using timedependent laxative use from all medication inventories collected during follow-up time [Additional file 1: Table S2]. Data on laxative use were updated at year 3 in the OS, and at years 1, 3, 6 and 9 in the CT. Follow-up time was censored three years after last medication collection. Multivariate linear regression methods were constructed to assess the association of baseline BMD for total hip, total spine and total body levels with any laxative use, as well as 3-year changes in BMD [Table 4]. For evaluating the relation of the duration of laxative intake to the incidence of falls and fractures, we used proportional hazards models to examine the association of different duration of laxative use ( $<1$ year, 1-3 years, or $>3 y e a r s)$ with the outcomes of interest [Table 5]. We also conducted selected subgroup analyses for associations of laxative use on the outcomes by age group, current hormone replacement therapy use, current psychoactive therapy, BMI and history of fracture [Additional file 1: Table S3]. Finally, to further explore laxative intake under randomized trial conditions we conducted subgroup analyses relating any laxative use to incidence of fracture and falls in the WHI Hormone Therapy Trial [Additional file 1: Table S4]. All analyses were conducted using SAS statistical software (version 9.2; SAS Institute Inc, Cary, North Carolina).

\section{Results}

Among the 161,808 women in the WHI OS and CTs, 160,404 had no prior hip fracture at baseline and were included in this study; 8907 (5.6\%) of study participants were currently using laxatives at baseline [Table 1]. Of those, 1603 persons used an oral stimulant containing laxative, 5372 a fiber containing laxative, 281 an osmotic containing laxative, 2525 a stool softener containing laxative and 33 a lubricant containing laxative [Table 2]. Nearly 4000 women (43.7\%) had used laxative medication for more than 3 years, 3091 (34.7\%) for 1 to 3 years, and 1924 (21.6\%) for less than 1 year [Table 5]. Those using laxatives at baseline were of older age, had lower BMI, more likely to have a lower physical functioning or a history of fractures, more likely to report low or fair health or osteoporosis, and to be included in the observational study (WHI-OS) [Table 1]. Concurrent use of psychoactive medications and hormone replacement therapy was more common among laxative users. Calcium and Vitamin D supplementation as well as bisphosphonates and calcitonin were more often used by laxative users. After adjustment for age, ethnicity and BMI laxative users tended to have a higher lumbar spine and total BMD. Other baseline characteristics were similar.

During 6.4 follow-up years/person in the laxative nonuser group $(n=151,497)$ as compared to 6.1 follow-up years/person in the user group $(n=8907), 50,175$ falls were reported in the non-user and 3484 falls in the usergroup [Table 2]. The age-adjusted rates of falls were $52.84 / 1000$ person-years for non-users and 63.35/1000 person-years for laxative users. Similarly, the rates of hip fractures were $1.54 / 1000$ and $1.75 / 1000$ and the rates of total fractures were $20.89 / 1000$ and $23.07 / 1000$ for laxative non-users and users [Additional file 1: Table S1]. 
Table 1 Baseline differences between users and non-users of laxatives

\begin{tabular}{|c|c|c|c|}
\hline & $\begin{array}{l}\text { Laxative } \\
\text { user } \\
(\mathrm{N}=8907)\end{array}$ & $\begin{array}{l}\text { Nonuser } \\
\text { of laxatives } \\
(\mathrm{N}=151,497)\end{array}$ & P-value \\
\hline \multicolumn{4}{|l|}{ Age } \\
\hline$<60$ & - & $51188(33.8 \%)$ & $<0.0001$ \\
\hline$>=60$ & 6745 (75.7\%) & 100309 (66.2\%) & \\
\hline
\end{tabular}

Race/ethnicity

$$
\begin{aligned}
& \text { White } \\
& \text { Black }
\end{aligned}
$$

Other

BMI (kg/m2)

$$
\begin{aligned}
& <25 \\
& 25-<30
\end{aligned}
$$$$
>=30
$$

Smoking

Never
Past

Current

> 7 Alcoholic drinks/week

\section{Nondrinker}

No

Yes

Coffee intake

$$
\begin{aligned}
& 0 \text { cups a day } \\
& <=1 \text { cup a day } \\
& 1-3 \text { cups a day } \\
& >4 \text { cups a day }
\end{aligned}
$$

Diabetes

$$
\text { No }
$$$$
\text { Yes }
$$

Physical activity (METs) h/wk

$$
\begin{aligned}
& 0, \text { Inactive } \\
& <5 \\
& 5-12 \\
& >=12
\end{aligned}
$$

Last medical visit within one year

$$
\begin{aligned}
& \text { No } \\
& \text { Yes }
\end{aligned}
$$

Fair or poor self-reported health

$$
\text { No }
$$$$
\text { Yes }
$$

Osteoporosis

$$
\text { No }
$$$$
\text { Yes }
$$

\section{5 (75.7\%) 100309 (66.2\%)}

$$
\begin{aligned}
& 7859(88.2 \%) \quad 124468(82.2 \%) \quad<0.0001 \\
& 630(7.1 \%) \quad 13899(9.2 \%)
\end{aligned}
$$$$
418(4.7 \%) \quad 13130(8.7 \%)
$$

$\begin{array}{lll}3334(37.7 \%) & 52350(34.9 \%) & <0.0001 \\ 3079(34.8 \%) & 52168(34.7 \%) & \\ 2433(27.5 \%) & 45624(30.4 \%)\end{array}$

$2433(27.5 \%) \quad 45624$ (30.4\%)

$4353(49.5 \%) \quad 76367(51.1 \%) \quad<0.0001$

3995 (45.4\%)

$$
451(5.1 \%) \quad 10581(7.1 \%)
$$

3885 (43.7\%)

$1026(11.6 \%)$

$63883(42.3 \%) \quad 0.0252$

$69542(46.1 \%)$

$17568(11.6 \%)$

3977 (45.1\%)

$1428(16.2 \%)$

$2567(29.1 \%)$

$848(9.6 \%)$

$61373(40.9 \%)<0.0001$

$23379(15.6 \%)$

47787 (31.9\%)

$17359(11.6 \%)$

$$
\begin{array}{rrr}
8482(95.3 \%) & 144679(95.6 \%) & 0.2068 \\
418(4.7 \%) & 6680(4.4 \%) &
\end{array}
$$

$1259(14.6 \%)$

$2083(24.1 \%)$

$3584(41.5 \%)$

$22956(15.9 \%)<0.0001$

$30445(21.1 \%)$

$34366(23.8 \%)$

56535 (39.2\%)

$$
997(11.5 \%) \quad 26958(18.4 \%) \quad<0.0001
$$$$
7683(88.5 \%) \quad 119360(81.6 \%)
$$

$$
7738(87.4 \%) \quad 137171(91.1 \%) \quad<0.0001
$$$$
1119(12.6 \%) \quad 13339(8.9 \%)
$$

$$
\begin{aligned}
& 7881(89.4 \%) \quad 138322(92.7 \%) \quad<0.0001 \\
& 932(10.6 \%) \quad 10972(7.3 \%)
\end{aligned}
$$

$3972(44.7 \%)$

$1712(19.8 \%)$
Table 1 Baseline differences between users and non-users of laxatives (Continued)

\begin{tabular}{crrr}
\hline $\begin{array}{c}\text { Physical functioning }>90 \\
\text { No }\end{array}$ & & & \\
Yes & $2567(29.3 \%)$ & $55808(37.6 \%)$ & \\
Number of chronic conditions $^{a}$ & & & \\
0 & $1771(19.9 \%)$ & $43185(28.5 \%)$ & $<0.0001$ \\
1 & $3016(33.9 \%)$ & $53641(35.4 \%)$ & \\
2 & $2393(26.9 \%)$ & $34652(22.9 \%)$ & \\
3 & $1226(13.8 \%)$ & $14609(9.6 \%)$ \\
$>=4$ & $501(5.6 \%)$ & $5410(3.6 \%)$ &
\end{tabular}

History of falls

No
Yes

7702 (86.5\%)

$133542(88.1 \%)<0.0001$

$1205(13.5 \%) \quad 17955(11.9 \%)$

Fracture on or after age 55

$\begin{array}{llll}\text { No } & 5818(81.9 \%) & 96946(84.3 \%) & <0.0001 \\ \text { Yes } & 1290(18.1 \%) & 17991(15.7 \%)\end{array}$

Hormone therapy use

Never used

$3233(36.3 \%)$

$66953(44.2 \%)<0.0001$

Past user

Current user

$1692(19.0 \%)$

24005 (15.9\%)

3977 (44.7\%)

60405 (39.9\%)

Bisphosphonates use

No

$8679(97.4 \%)$

$148671(98.1 \%)<0.0001$

Yes

$228(2.6 \%)$

Corticosteroid use

No

Yes

8813 (98.9\%)

2824 (1.9\%)

$$
94(1.1 \%)
$$

$150222(99.2 \%)$

0.0319

Calcitonin use

$$
\text { No }
$$

Yes

8861 (99.5\%)

$151077(99.7 \%)<0.0001$

$46(0.5 \%)$

$418(0.3 \%)$

Psychoactive drug use

No

Yes

$7123(80.0 \%)$

$1748(20.0 \%)$

$136454(90.1 \%)<0.0001$

Total baseline calcium

intake $(\mathrm{mg} / \mathrm{d})$

$<400$
$400-<800$
$800-<1200$
$>=1200$

Calcium supplement use

$\begin{array}{llll}\text { No } & 2974(33.4 \%) & 68257(45.1 \%) & <0.0001 \\ \text { Yes } & 5933(66.6 \%) & 83238(54.9 \%) & \end{array}$

Total baseline Vitamin D intake (IU/d)

$\begin{array}{llll}<200 & 2557(29.5 \%) & 56042(38.1 \%) & <0.0001 \\ 200-<400 & 1457(16.8 \%) & 27342(18.6 \%) \\ 400-<600 & 2440(28.2 \%) & 35784(24.4 \%) \\ >=600 & 2201(25.4 \%) & 27742(18.9 \%)\end{array}$


Table 1 Baseline differences between users and non-users of laxatives (Continued)

\begin{tabular}{|c|c|c|c|}
\hline \multicolumn{4}{|l|}{ Vitamin D supplement use } \\
\hline No & $3647(40.9 \%)$ & $80042(52.8 \%)$ & $<0.0001$ \\
\hline Yes & $5260(59.1 \%)$ & $71453(47.2 \%)$ & \\
\hline $\begin{array}{l}\text { Baseline total body } \\
\text { bone density }{ }^{b}\end{array}$ & 1.017 & 1.014 & 0.5236 \\
\hline $\begin{array}{l}\text { Baseline total body } \\
\text { bone density adjusted }^{c}\end{array}$ & 1.027 & 1.012 & 0.0017 \\
\hline $\begin{array}{l}\text { Baseline lumbar spine } \\
\text { bone density }{ }^{\mathrm{b}}\end{array}$ & 0.990 & 0.979 & 0.1551 \\
\hline $\begin{array}{l}\text { Baseline lumbar spine } \\
\text { bone density adjusted }^{c}\end{array}$ & 0.998 & 0.978 & 0.0065 \\
\hline $\begin{array}{l}\text { Baseline total hip } \\
\text { bone density }\end{array}$ & 0.837 & 0.853 & 0.0123 \\
\hline $\begin{array}{l}\text { Baseline total hip } \\
\text { bone density adjusted }^{c}\end{array}$ & 0.850 & 0.850 & 0.9395 \\
\hline \multicolumn{4}{|l|}{ Hormone therapy trial } \\
\hline No & $7694(86.4 \%)$ & $125585(82.9 \%)$ & $<0.0001$ \\
\hline Yes & $1213(13.6 \%)$ & $25912(17.1 \%)$ & \\
\hline \multicolumn{4}{|l|}{ Dietary modification trial } \\
\hline No & $6652(74.7 \%)$ & $105272(69.5 \%)$ & $<0.0001$ \\
\hline Yes & $2255(25.3 \%)$ & $46225(30.5 \%)$ & \\
\hline \multicolumn{4}{|l|}{ Calcium Vitamin D trial } \\
\hline No & $7435(83.5 \%)$ & $116920(77.2 \%)$ & $<0.0001$ \\
\hline Yes & $1472(16.5 \%)$ & $34577(22.8 \%)$ & \\
\hline \multicolumn{4}{|l|}{ Observational study } \\
\hline No & $3168(35.6 \%)$ & $64447(42.5 \%)$ & $<0.0001$ \\
\hline Yes & $5739(64.4 \%)$ & $87050(57.5 \%)$ & \\
\hline
\end{tabular}

${ }^{a}$ Includes treated diabetes mellitus, stroke, any cancer, CVD, arthritis, hypertension, 2 or more falls, emphysema.

b From subset.

c Adjusted for age, ethnicity, and BMI.

In minimally adjusted models a significant association was found between 'any laxative use' and falls (HR, 1.21; 95\% CI, 1.17-1.25), and total fractures (HR, 1.07; 95\% CI, 1.01-1.13) at baseline, while the association with hip fractures was not statistically significant (HR, 1.11; 95\% CI, 0.94-1.32) [Table 3].When stratifying these associations by the various laxative subgroups, we noted 'oral stimulant', 'fiber' and 'stool softener containing laxatives' to be significantly associated with the risk of falls. After full adjustment 'any laxative use' (Model 2A: HR, 1.06; 95\% CI, 1.03-1.10), 'fiber containing laxatives' (Model 2A: HR, 1.06; 95\% CI, 1.01-1.11) and 'stool softener containing laxatives' (Model 2A: HR, 1.08; 95\% CI, 1.021.15) remained statistically significantly associated with falls. These results did only marginally change after additional adjustment for history of falls. No significant relationship between laxative use and the risk for fractures was found after fully accounting for confounding factors. To investigate the effects of laxative use over time, we additionally constructed time-dependent models [Additional file 1: Table S2]. There we continued to find a significant association between the risk for falls and 'any laxative use' (HR, 1.06; 95\% CI, 1.02-1.10) and 'stool softener containing laxatives' (HR 1.13; 95\% CI, 1.061.21). In contrast to baseline analyses, 'oral stimulant containing laxatives' (HR, 2.04; 95\% CI, 1.36-3.07) and 'stool softener containing laxatives' (HR, 1.50; 95\% CI, 1.09-2.05) remained significantly associated with the risk for hip fractures even after full adjustment. No relationship between the risk for total fractures and any laxative subgroup except for 'stool softener containing laxatives' (HR, 1.12; 95\% CI, 1.00-1.25) could be established in time-dependent analyses.

At baseline, women using laxatives had similar BMD at the hip level (adjusted mean, 0.85 vs. $0.84 \mathrm{~g} / \mathrm{cm}^{2}$ ) compared with nonusers. Total body BMD and total spine BMD differed significantly in users compared with nonusers (adjusted mean, 1.02 vs. $1.01 \mathrm{~g} / \mathrm{cm}^{2}$ and 0.99 vs. $0.98 \mathrm{~g} / \mathrm{cm}^{2}$ ) [Table 4]. Examining the 3-year BMD change from baseline, we found small statistically nonsignificant increases in BMD in all areas of interest, which may stem from the fact that a high proportion of women in both groups were part of the active treatment arms in the WHI-CT involving calcium and vitamin D supplementation and/or hormone trial (HT).

Any laxative use tended to be associated with an increased hazard for falls in stratified analysis by exposure duration while no clear trend could be observed for the risk for hip fractures or total fractures [Table 5]. Because laxative use may have different effects on various subpopulations, we additional undertook several subgroup analyses $[28,59,60]$. Laxative use was associated with an increased hazard for total fractures by age in women older than 65 years (HR, 1.06; 95\% CI, 0.99-1.14, interaction $\mathrm{p}$-value $=0.05)$, although no significant interaction by age was found for falls and hip fractures. Psychoactive therapy was associated with a lower risk for total fractures (HR, 0.91; 95\% CI, 0.81-1.03, interaction p-value $=0.05$ ), whereas no significant influence on falls or hip fractures was noted [Additional file 1: Table S3]. Finally, we found no significant difference in the prevalence of laxative intake between women receiving hormone therapy or placebo under WHI Hormone Therapy Trial settings [Additional file 1: Table S4]. Interaction tests for women stratified by trial randomization did not result in significant differences in risk of falls and fractures between laxative users and non-users.

\section{Discussion}

In this large prospective study of postmenopausal women with no prior hip fracture, we found a statistically significant association of laxative use with the occurrence of falls $(\geq 2)$. Laxative use was not associated with an increased 
Table 2 Clinical outcomes according to laxative use and drug class

\begin{tabular}{|c|c|c|c|c|c|c|c|}
\hline Clinical outcome & $\begin{array}{l}\text { No Laxative use } \\
(\mathrm{N}=151497)\end{array}$ & $\begin{array}{l}\text { Laxative use } \\
(\mathrm{N}=8907)\end{array}$ & $\begin{array}{l}\text { Oral } \\
\text { stimulant } \\
\text { containing } \\
\text { laxative } \\
(\mathrm{N}=1603) \\
\end{array}$ & $\begin{array}{l}\text { Fiber } \\
\text { containing } \\
\text { laxative } \\
(\mathrm{N}=5372)\end{array}$ & $\begin{array}{l}\text { Osmotic } \\
\text { containing } \\
\text { laxative } \\
(\mathrm{N}=281)\end{array}$ & $\begin{array}{l}\text { Stool softener } \\
\text { containing } \\
\text { laxative } \\
(\mathrm{N}=2525)\end{array}$ & $\begin{array}{l}\text { Lubricant } \\
\text { containing } \\
\text { laxative } \\
(\mathrm{N}=33)\end{array}$ \\
\hline Falls $(>2)$ & 50175 (33.9\%) & $3484(40.0 \%)$ & $588(37.7 \%)$ & $2040(38.8 \%)$ & $106(39.3 \%)$ & $1067(43.3 \%)$ & $15(46.9 \%)$ \\
\hline p-yrs & 959,729 & 53,592 & & & & & \\
\hline Follow-up yrs (SD) / person & $6.4(2.7)$ & $6.1(2.7)$ & & & & & \\
\hline Hip fracture & $1706(1.1 \%)$ & $142(1.6 \%)$ & $33(2.1 \%)$ & $73(1.4 \%)$ & $4(1.4 \%)$ & $52(2.1 \%)$ & $0(0.0 \%)$ \\
\hline p-yrs & $1,171,740$ & 67,909 & & & & & \\
\hline Follow-up yrs (SD) / person & $7.8(1.6)$ & $7.6(1.7)$ & & & & & \\
\hline Total fracture & $21642(14.3 \%)$ & $1476(16.6 \%)$ & $255(15.9 \%)$ & $852(15.9 \%)$ & $48(17.1 \%)$ & 449 (17.8\%) & $6(18.2 \%)$ \\
\hline p-yrs & $1,053,650$ & 61,029 & & & & & \\
\hline Follow-up yrs (SD) / person & $7.2(2.2)$ & $7.1(2.2)$ & & & & & \\
\hline
\end{tabular}

risk for total fractures and hip fractures after fully adjustment for confounding factors. Among laxative users at baseline, the HR for falls was 1.06, for hip fractures 1.02 and for total fractures 1.01. The CIs indicate that the true HR for falls may be as low as 1.03 or as high as 1.10 , for hip fractures 0.85 and 1.22 and for total fractures 0.96 and
1.07 respectively. Using time-dependent analyses laxative use remained statistically significantly associated with an increased risk for falls. These results were most likely driven by a higher number of women starting to take laxatives during follow-up; thus we achieved a higher power to detect differences between users and non-users.

Table 3 Adjusted hazards ratio of fractures and falls by laxative use at baseline

\begin{tabular}{|c|c|c|c|c|c|c|c|}
\hline & & $\begin{array}{l}\text { Model } 1 \\
\text { HR }(95 \% \mathrm{Cl})^{*}\end{array}$ & $\begin{array}{l}\text { Model } 1 \\
\text { p-value* }\end{array}$ & $\begin{array}{l}\text { Model 2A } \\
\text { HR }(95 \% \mathrm{Cl})^{* *}\end{array}$ & $\begin{array}{l}\text { Model 2A } \\
\text { p-value** }\end{array}$ & $\begin{array}{l}\text { Model 2B } \\
\text { HR }(95 \% \mathrm{CI})^{* *}\end{array}$ & $\begin{array}{l}\text { Model 2B } \\
\text { p-value*** }\end{array}$ \\
\hline \multirow[t]{6}{*}{ Falls $(\geq 2)$} & Any laxative use & $1.21(1.17,1.25)$ & $<.0001$ & $1.06(1.03,1.10)$ & 0.0006 & $1.06(1.03,1.10)$ & 0.0008 \\
\hline & Oral stimulant containing laxative & $1.15(1.06,1.25)$ & 0.0009 & $0.98(0.90,1.07)$ & 0.6162 & $0.97(0.89,1.05)$ & 0.4450 \\
\hline & Fiber containing laxative & $1.15(1.10,1.20)$ & $<.0001$ & $1.06(1.01,1.11)$ & 0.0147 & $1.05(1.01,1.10)$ & 0.0272 \\
\hline & Osmotic containing laxative & $1.20(0.99,1.46)$ & 0.0577 & $1.10(0.90,1.34)$ & 0.3493 & $1.10(0.90,1.34)$ & 0.3510 \\
\hline & Stool softener containing laxative & $1.35(1.27,1.44)$ & $<.0001$ & $1.08(1.02,1.15)$ & 0.0145 & $1.10(1.03,1.17)$ & 0.0036 \\
\hline & Lubricant containing laxative & $1.51(0.91,2.50)$ & 0.1113 & $1.10(0.63,1.94)$ & 0.7390 & $1.06(0.60,1.87)$ & 0.8377 \\
\hline \multirow[t]{6}{*}{ Hip fracture } & Any laxative use & $1.11(0.94,1.32)$ & 0.2200 & $1.02(0.85,1.22)$ & 0.8509 & $1.02(0.85,1.22)$ & 0.8426 \\
\hline & Oral stimulant containing laxative & $1.62(1.15,2.28)$ & 0.0062 & $1.31(0.90,1.91)$ & 0.1556 & $1.32(0.91,1.93)$ & 0.1444 \\
\hline & Fiber containing laxative & $0.92(0.73,1.16)$ & 0.4907 & $0.86(0.67,1.11)$ & 0.2514 & $0.87(0.67,1.11)$ & 0.2552 \\
\hline & Osmotic containing laxative & $1.06(0.40,2.82)$ & 0.9118 & $0.98(0.37,2.61)$ & 0.9625 & $1.00(0.38,2.68)$ & 0.9942 \\
\hline & Stool softener containing laxative & $1.46(1.11,1.93)$ & 0.0070 & $1.26(0.94,1.69)$ & 0.1203 & $1.26(0.94,1.69)$ & 0.1273 \\
\hline & Lubricant containing laxative & $N A^{* * *}$ & & & & & \\
\hline \multirow[t]{6}{*}{ Total fracture } & Any laxative use & $1.07(1.01,1.13)$ & 0.0137 & $1.01(0.96,1.07)$ & 0.6237 & $1.02(0.96,1.07)$ & 0.5511 \\
\hline & Oral stimulant containing laxative & $1.13(0.99,1.27)$ & 0.0606 & $1.04(0.92,1.18)$ & 0.5391 & $1.04(0.91,1.18)$ & 0.5725 \\
\hline & Fiber containing laxative & $0.99(0.92,1.06)$ & 0.6831 & $0.97(0.90,1.04)$ & 0.4089 & $0.97(0.90,1.04)$ & 0.4004 \\
\hline & Osmotic containing laxative & $1.15(0.87,1.53)$ & 0.3263 & $1.10(0.82,1.47)$ & 0.5285 & $1.12(0.84,1.49)$ & 0.4517 \\
\hline & Stool softener containing laxative & $1.20(1.09,1.31)$ & 0.0002 & $1.05(0.95,1.16)$ & 0.3285 & $1.06(0.96,1.17)$ & 0.2331 \\
\hline & Lubricant containing laxative & $1.29(0.58,2.88)$ & 0.5267 & $1.29(0.58,2.87)$ & 0.5322 & $1.26(0.57,2.81)$ & 0.5665 \\
\hline
\end{tabular}

* Adjusted for age, ethnicity, and WHI clinical trial indicators.

** Adjusted for age, ethnicity, BMI, WHI clinical trial indicators, smoking status, physical activity, self-reported health, treated diabetes, history of fracture after age 55 , corticosteroid use, physical function score, number of chronic medical conditions (including treated diabetes mellitus, stroke, any cancer, CVD, arthritis, hypertension, and emphysema), number of psychoactive drugs (including antipsychotic, antiepileptic, anxiolytic, hypnotic and antidepressant drug use), use of HRT, and bisphosphonate use, and in Model 2B history of falls $(\geq 2)$.

*** No hip fractures occurred in this group. 
Table 4 Three-year changes in mean bone mineral density (BMD) according to laxative use

\begin{tabular}{|c|c|c|c|c|c|c|c|}
\hline & \multicolumn{2}{|c|}{ Laxative Use } & \multicolumn{2}{|c|}{ No Laxative Use } & \multirow[b]{2}{*}{ P-value * } & \multirow[b]{2}{*}{ P-value ** } & \multirow[b]{2}{*}{ P-value **- } \\
\hline & $\mathbf{N}$ & Mean (SE) * & $N$ & Mean $(\mathrm{SE})$ * & & & \\
\hline \multicolumn{8}{|l|}{ Total hip } \\
\hline Baseline & 509 & $0.842(0.006)$ & 10791 & $0.847(0.001)$ & 0.4170 & 0.8376 & 0.8624 \\
\hline Year 3 & 424 & $0.847(0.006)$ & 8916 & $0.854(0.001)$ & 0.2767 & 0.3282 & 0.3409 \\
\hline Absolute change & 422 & $0.004(0.002)$ & 8810 & $0.006(0.000)$ & 0.3866 & 0.3734 & 0.3714 \\
\hline Percent change & 422 & $0.596(0.201)$ & 8810 & $0.730(0.047)$ & 0.5108 & 0.4856 & 0.4791 \\
\hline \multicolumn{8}{|l|}{ Total spine } \\
\hline Baseline & 487 & $0.991(0.007)$ & 10456 & $0.975(0.002)$ & 0.0339 & 0.1307 & 0.1325 \\
\hline Year 3 & 401 & $1.010(0.009)$ & 8653 & $0.995(0.002)$ & 0.0836 & 0.4034 & 0.3974 \\
\hline Absolute change & 400 & $0.017(0.002)$ & 8579 & $0.021(0.001)$ & 0.2075 & 0.1396 & 0.1573 \\
\hline Percent change & 400 & $1.840(0.257)$ & 8579 & $2.198(0.060)$ & 0.1711 & 0.1324 & 0.1505 \\
\hline \multicolumn{8}{|l|}{ Total body } \\
\hline Baseline & 512 & $1.023(0.004)$ & 10794 & $1.011(0.001)$ & 0.0090 & 0.0209 & 0.0234 \\
\hline Year 3 & 424 & $1.033(0.005)$ & 8891 & $1.023(0.001)$ & 0.0692 & 0.1754 & 0.1854 \\
\hline Absolute change & 423 & $0.012(0.002)$ & 8796 & $0.011(0.000)$ & 0.6686 & 0.9132 & 0.8580 \\
\hline Percent change & 423 & $1.236(0.181)$ & 8796 & $1.150(0.043)$ & 0.6422 & 0.8545 & 0.7946 \\
\hline
\end{tabular}

* Adjusted for age, ethnicity, and WHI clinical trial indicators.

** Adjusted for age, ethnicity, BMI, WHI clinical trial indicators, smoking status, physical activity, self-reported health, treated diabetes, history of fracture after age 55 , corticosteroid use, physical function score, number of chronic medical conditions (including treated diabetes mellitus, stroke, any cancer, CVD, arthritis, hypertension, and emphysema), number of psychoactive drugs (including antipsychotic, antiepileptic, anxiolytic, hypnotic and antidepressant drug use), use of HRT, and bisphosphonate use.

*** Adjusted as in $\left(^{* *}\right)$ with the addition of history of falls $(\geq 2)$.

Nonetheless, we cannot rule out residual confounding by indication as having partly influenced our results. Longer duration of laxative use did not show a statistically significant association with the risk for hip fractures or total fractures, while a positive relationship for falls could be observed.

Several intrinsic and extrinsic effects of laxatives have been associated with their use providing potential

Table 5 Adjusted hazards ratio of fractures and falls by any laxative use duration

\begin{tabular}{|c|c|c|c|c|}
\hline \multirow{2}{*}{$\begin{array}{l}\text { Any } \\
\text { laxative } \\
\text { use }\end{array}$} & \multirow[t]{2}{*}{$\mathbf{N}$} & \multicolumn{3}{|c|}{$\operatorname{HR}(95 \% \mathrm{Cl})^{* *}$} \\
\hline & & Falls $(>2)$ & Hip fracture & Total fracture \\
\hline Nonuser & 151,497 & 1 & 1 & 1 \\
\hline$<1$ year & 1924 & $1.11(1.04,1.74)$ & $1.17(0.89,1.55)$ & $1.06(0.97,1.16)$ \\
\hline $1-3$ years & 3091 & $1.07(0.99,1.15)$ & $1.02(0.69,1.50)$ & $1.03(0.92,1.15)$ \\
\hline$>3$ years & 3892 & $1.00(0.95,1.06)$ & $0.90(0.68,1.19)$ & $0.97(0.89,1.05)$ \\
\hline $\begin{array}{l}p \text {-value } \\
\text { for linear } \\
\text { trend }\end{array}$ & & 0.0458 & 0.9553 & 0.9996 \\
\hline
\end{tabular}

** Adjusted for age, ethnicity, BMI, WHI clinical trial indicators, smoking status, physical activity, self-reported health, treated diabetes, history of fracture after age 55, corticosteroid use, physical function score, number of chronic medical conditions (including treated diabetes mellitus, stroke, any cancer, CVD, arthritis, hypertension, 2 or more falls and emphysema), number of psychoactive drugs (including antipsychotic, antiepileptic, anxiolytic, hypnotic and antidepressant drug use), use of HRT, and bisphosphonate use. mechanisms for an altered risk for falls and bone outcomes $[2,5,9,25,28,61]$. Extrinsically, laxatives can increase the urgency to void with resulting higher risk of falling. Intrinsically, laxatives can lead to diarrhoea with associated electrolyte and fluid imbalances. Potassium is the main electrolyte being lost followed by reductions in sodium and chloride. Generalized muscle weakness, lassitude and cardiac arrhythmias may be a direct consequence of electrolyte disturbances resulting in an increased risk for falls and bone outcomes [8-14,32,35-38]. Most recent reports have linked electrolyte imbalances such as mild hyponatremia to osteoporosis and fracture risk [9-11,30,61-63]. Additionally, laxatives have also been described to irritate bowel functioning and bowel disturbances in the colon and small bowl $[2,3,28]$. Fat-soluble vitamin malabsorption secondary to these types of laxative induced side effects of small intestine damage may further predispose for falls, fractures and BMD loss [13,14,32-35,37]. Indeed, prior epidemiological studies have shown that laxative use is associated with an increased risk for falls [2,20-28]. One of the first was the St. Louis OASIS study in which laxative use was found to be an important risk factor for multiple falls in elderly people living in the community (OR, 2.14; 95\% CI, 1.02-4.49) [59]. Other cross-sectionally designed studies confirmed this positive association [22,23,27]. Observational studies examining medications and chronic diseases with one year 
follow-up period reported risks for falling between OR 1.09 (95\% CI, 0.86-1.38) and 2.51 (95\% CI, 1.50-4.22) with laxative use [22,23,25,26,29]. A recent meta-analysis summarizing the risks for falls related to laxatives elderly individuals resulted in an OR 2.03 (95\% CI, 1.52-2.72) [28]. Nevertheless, external validity of many included studies may be limited as the mean age of the study population was above 80 years old [20,22,27,29] with a small number of participants, events and short follow-up [20,22]. Last, data on fracture risk or change in BMD were not assessed even though a history of falls is an independent risk factor for fractures [39].

In contrast to previously published findings [28,59], our results indicate a modest association between laxative use and the occurrence of falls, whereas we found no relationship between laxative use and fractures. Furthermore, although baseline total body bone density and total lumbar spine density varied between laxative users or non-users, three-year changes in BMD did not show any significant differences between both groups for all BMD areas. In particular, we did not observe differences in total hip bone density between laxative users or not users. These findings are surprising given the different baseline characteristics of laxative users and non-users suggesting that laxative users have a higher number of chronic conditions, lower physical functioning and are of older age, factors known to influence the risk for falls, fractures and changes in BMD $[26,64,65]$. It may be explained by various levels of calcium and vitamin D intake between the two groups. Nevertheless, given our hypothesis that laxative intake may potentially cause electrolyte imbalances, vitamin D malabsorption or even small intestine mucosal damage, we expected a strong relationship between falls and laxative use and an increased risk for fractures and BMD reductions. To further investigate the association of laxative use with the incidence of falls and fractures when used concomitantly with certain medications (psychoactive drugs, hormone therapy), in certain age groups, with different BMI levels or history of fracture, we conducted a subgroup analysis. We found a slightly higher risk for falls in laxative users being of older age (>65 y), while current psychoactive therapy, BMI, history of fracture had no negative effect. Moreover, we noted a higher risk for falls in laxative users under current hormone treatment in the total study population (WHI OS and CTs) which we and others did not continue to observe in a hormone trial setting [66]. On the other hand, older women using laxatives showed a significant risk for total fractures despite similar BMD levels. Finally, concomitant use of psychoactive agents did not increase the risk for hip and total fractures in our study population.

The strengths of our analysis include the sample size of our study population, the large number of complete follow- up and the structured availability of several confounding factors. Nonetheless, our study also exhibits several limitations. Among those, first the relative low prevalence of long time laxative use and the missing data on dosing may limit the validity of this study. Also, since many commercially available laxatives are mixture products consisting of two or more pharmacologically active substances, our analyses on the various categories of laxatives are limited due to missing information on the exact composition of these products. Second, laxative intake as well as incident falls and fractures were ascertained via self-report. Hence, we cannot exclude information bias. However, since laxative intake is likely to be assumed to be underreported, the actual effects may be underestimated. Third, even though a wide range of confounding factors was considered in the analysis, residual confounding cannot be excluded. Our results may be partly confounded by indication for which we controlled by measures of restriction, stratification and extensive multivariate adjustment. Additionally, even though this study was designed prospectively, reverse causation may have influenced our results. Last, as our study population consisted of postmenopausal women, generalizability may be limited and further research in other study populations is warranted.

\section{Conclusions}

Laxative users are characterized by older age, lower physical functioning, more comorbidities and higher concurrent use of psychoactive medications and hormone replacement therapy. Our findings support an association between any laxative use and increased risk for falls but not with total fracture risk or hip fracture risk. A relationship of laxative use with changes in BMD over time was not demonstrated, but calcium and vitamin D were more often supplemented by laxative users. Bisphosphonate and calcitonin use was more likely in this group as well. Thus, questions remain regarding the influence on bone health and potential risks associated with long-term laxative use. Given the low cost, widespread use and over-the-counter availability it appears prudent to periodically reevaluate laxative use. Maintaining physical functioning, and providing adequate treatment of comorbidities that predispose individuals for falls should be considered as first measures to avoid potential negative consequences associated with laxative use.

\section{Additional file}

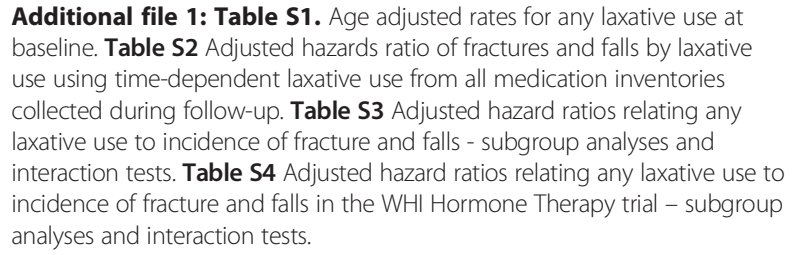

Additional file 1: Table S1. Age adjusted rates for any laxative use at baseline. Table S2 Adjusted hazards ratio of fractures and falls by laxative use using time-dependent laxative use from all medication inventories collected during follow-up. Table S3 Adjusted hazard ratios relating any laxative use to incidence of fracture and falls - subgroup analyses and interaction tests. Table S4 Adjusted hazard ratios relating any laxative use to incidence of fracture and falls in the WHI Hormone Therapy trial - subgroup analyses and interaction tests. 


\section{Competing interests}

Dr. Carnahan's work was supported by the University of lowa Institute for Clinical and Translational Science, and an Agency for Healthcare Research and Quality Centers for Education and Research on Therapeutics cooperative agreement \#5 U18 HS016094 (the lowa Older Adults CERT). In the past 5 years he received research funding from Boehringer Ingelheim, Forest Laboratories, Wyeth, and Ortho-McNeil. None of the other authors reported disclosures.

\section{Authors' contributions}

MP and $\mathrm{BH}$ had full access to all of the data in the study and take responsibility for the integrity of the data and the accuracy of the data analysis. Study concept and design: BH, SWW Acquisition of data: BP, MP, SWW Analysis and interpretation of data: MP, BH, WvB, JWB, JWW, RMC, RBW Drafting of the manuscript: $B H, J W B, J W W, J O, R B W$ Critical revision of the manuscript for important intellectual content: JWB, RCM, JO, RCM, RBW SWW, WVB Statistical analysis: MP Obtained funding: SWW Administrative, technical, or material support: SWW Study supervision: SWW. All authors read and approved the final manuscript.

\section{Acknowledgments}

WHI Short List of Investigators: Program Office: (National Heart, Lung, and Blood Institute, Bethesda, Maryland) Jacques Rossouw, Shari Ludlam, Dale Burwen, Joan McGowan, Leslie Ford, and Nancy Geller Clinical Coordinating Center: Clinical Coordinating Center: (Fred Hutchinson Cancer Research Center, Seattle, WA) Garnet Anderson, Ross Prentice, Andrea LaCroix, and Charles Kooperberg. Investigators and Academic Centers: (Brigham and Women's Hospital, Harvard Medical School, Boston, MA) JoAnn E. Manson (MedStar Health Research Institute/Howard University, Washington, DC) Barbara V. Howard; (Stanford Prevention Research Center, Stanford, CA) Marcia L. Stefanick; (The Ohio State University, Columbus, OH) Rebecca Jackson; (University of Arizona, Tucson/Phoenix, AZ) Cynthia A. Thomson; (University at Buffalo, Buffalo, NY) Jean Wactawski-Wende; (University of Florida, Gainesville/Jacksonville, FL) Marian Limacher; (University of lowa, lowa City/Davenport, IA) Robert Wallace; (University of Pittsburgh, Pittsburgh, PA) Lewis Kuller; (Wake Forest University School of Medicine, Winston-Salem, NC) Sally Shumaker. Women's Health Initiative Memory Study: (Wake Forest University School of Medicine, Winston-Salem, NC) Sally Shumaker.

\section{Funding/support}

The WHI program is funded by the National Heart, Lung, and Blood Institute, National Institutes of Health, U.S. Department of Health and Human Services through contracts HHSN268201100046C, HHSN268201100001C, HHSN268201100002C, HHSN268201100003C, HHSN268201100004C, and HHSN271201100004C

\section{Author details}

${ }^{1}$ Department of Internal Medicine I, Comprehensive Heart Failure Center, University of Würzburg, Oberdürrbacher Strasse 6, 97080, Würzburg, Germany. ${ }^{2}$ Fred Hutchinson Cancer Research Center, Seattle, Washington, USA. ${ }^{3}$ Arizona Cancer Center, University of Arizona, Tucson, Arizona, USA. ${ }^{4}$ Department of Social and Preventive Medicine, University at Buffalo, SUNY School of Public Health and Health Professions, Buffalo, NY, USA. ${ }^{5}$ Department of Epidemiology, University of lowa College of Public Health, lowa City, lowa, USA. ${ }^{6}$ Division of Preventive and Behavioral Medicine, University of Massachusetts Medical School, Worcester, Massachusetts, USA. ${ }^{7}$ Department of Surgery \& Division of Cardiothoracic Surgery, Froedtert Memorial Hospital \& Medical College of Wisconsin, Milwaukee, WI, USA. ${ }^{8}$ Department of Epidemiology and Population Health, Albert Einstein College of Medicine, Bronx, New York, USA

Received: 14 August 2012 Accepted: 22 April 2013 Published: 1 May 2013

\section{References}

1. Consumer Health Care Association: OTC Sales by Category; 2011. [http://www. chpa-info.org/pressroom/Sales_Category.aspx] (Last accessed 03-25-2013.

2. Baker EH, Sandle Gl: Complications of laxative abuse. Annu Rev Med 1996, 47:127-134

3. Moriarty KJ, Silk DB: Laxative abuse. Dig Dis 1988, 6(1):15-29.
4. Roerig JL, Steffen KJ, Mitchell JE, Zunker C: Laxative abuse: epidemiology, diagnosis and management. Drugs 2010, 70(12):1487-1503.

5. Fleischer N, Brown H, Graham DY, Delena S: Chronic laxative-induced hyperaldosteronism and hypokalemia simulating Bartter's syndrome. Ann Intern Med 1969, 70(4):791-798

6. Sankar NS, Donaldson D: Lessons to be learned: a case study approach diuretic therapy and a laxative causing electrolyte and water imbalance, loss of attention, a fall and subsequent fractures of the tibia and fibula in an elderly lady. J R Soc Promot Health 1998, 118(4):237-240.

7. Yakabowich M: Prescribe with care. The role of laxatives in the treatment of constipation. J Gerontol Nurs 1990, 16(7):4-11.

8. Decaux G: Is asymptomatic hyponatremia really asymptomatic? Am J Med 2006, 119(7 Suppl 1):S79-82.

9. Gankam Kengne F, Andres C, Sattar L, Melot C, Decaux G: Mild hyponatremia and risk of fracture in the ambulatory elderly. QJM 2008, 101(7):583-588.

10. Kinsella S, Moran S, Sullivan MO, Molloy MG, Eustace JA: Hyponatremia independent of osteoporosis is associated with fracture occurrence. Clin J Am Soc Nephrol 2010, 5(2):275-280.

11. Sajadieh A, Binici Z, Mouridsen MR, Nielsen OW, Hansen JF, Haugaard SB: Mild hyponatremia carries a poor prognosis in community subjects. Am J Med 2009, 122(7):679-686.

12. Cranney A, Weiler HA, O'Donnell S, Puil L: Summary of evidence-based review on vitamin $D$ efficacy and safety in relation to bone health. Am J Clin Nutr 2008, 88(2):513S-519S.

13. Janssen HC, Samson MM, Verhaar HJ: Vitamin D deficiency, muscle function, and falls in elderly people. Am J Clin Nutr 2002, 75(4):611-615.

14. McCarroll KG, Robinson DJ, Coughlan A, Healy M, Kenny RA, Cunningham C: Vitamin D and orthostatic hypotension. Age Ageing 2012, 41(6):810-813.

15. Carbone LD, Johnson KC, Bush AJ, Robbins J, Larson JC, Thomas A, LaCroix $A Z$ : Loop diuretic use and fracture in postmenopausal women: findings from the Women's Health Initiative. Arch Intern Med 2009, 169(2):132-140.

16. Carbone LD, Johnson KC, Robbins J, Larson JC, Curb JD, Watson K, Gass M, Lacroix AZ: Antiepileptic drug use, falls, fractures, and BMD in postmenopausal women: findings from the women's health initiative (WHI). J Bone Miner Res 2010, 25(4):873-881.

17. Hix JK, Silver S, Sterns RH: Diuretic-associated hyponatremia. Semin Nephrol 2011, 31(6):553-566.

18. Rastogi D, Pelter MA, Deamer RL: Evaluations of hospitalizations associated with thiazide-associated hyponatremia. J Clin Hypertens (Greenwich) 2012, 14(3):158-164

19. Sonnenblick M, Friedlander Y, Rosin AJ: Diuretic-induced severe hyponatremia. Review and analysis of 129 reported patients. Chest 1993 103(2):601-606

20. Kallin K, Gustafson Y, Sandman PO, Karlsson S: Drugs and falls in older people in geriatric care settings. Aging Clin Exp Res 2004, 16(4):270-276.

21. Kallin $K$, Gustafson $Y$, Sandman $P O$, Karlsson S: Factors associated with falls among older, cognitively impaired people in geriatric care settings: a population-based study. Am J Geriatr Psychiatry 2005, 13(6):501-509.

22. Lundin-Olsson $L$, Jensen J, Nyberg $L$, Gustafson $Y$ : Predicting falls in residential care by a risk assessment tool, staff judgement, and history of falls. Aging Clin Exp Res 2003, 15(1):51-59.

23. Luukinen $H$, Koski K, Laippala P, Kivela SL: Predictors for recurrent falls among the home-dwelling elderly. Scand J Prim Health Care 1995, 13(4):294-299.

24. Luukinen H, Koski K, Laippala P, Kivela SL: Risk factors for recurrent falls in the elderly in long-term institutional care. Public Health 1995, 109(1):57-65.

25. Ryynanen OP, Kivela SL, Honkanen R, Laippala P, Saano V: Medications and chronic diseases as risk factors for falling injuries in the elderly. Scand J Soc Med 1993, 21(4):264-271.

26. Spector W, Shaffer T, Potter DE, Correa-de-Araujo R, Rhona Limcangco M: Risk factors associated with the occurrence of fractures in U.S. nursing homes: resident and facility characteristics and prescription medications. J Am Geriatr Soc 2007, 55(3):327-333.

27. Svensson ML, Rundgren A, Landahl S: Falls in 84- to 85-year-old people living at home. Accid Anal Prev 1992, 24(5):527-537.

28. Bloch F, Thibaud M, Dugue B, Breque C, Rigaud AS, Kemoun G: Laxatives as a risk factor for iatrogenic falls in elderly subjects: myth or reality? Drugs Aging 2010, 27(11):895-901. 
29. von Heideken WP, Gustafson Y, Kallin K, Jensen J, Lundin-Olsson L: Falls in very old people: the population-based Umea 85+ study in Sweden. Arch Gerontol Geriatr 2009, 49(3):390-396.

30. McPherson E, Dunsmuir RA: Hyponatraemia in hip fracture patients. Scott Med J 2002, 47(5):115-116

31. Ayus JC, Moritz ML: Bone disease as a new complication of hyponatremia: moving beyond brain injury. Clin J Am Soc Nephrol 2010, 5(2):167-168.

32. Bischoff-Ferrari HA, Dawson-Hughes B, Willett WC, Staehelin HB, Bazemore MG, Zee RY, Wong JB: Effect of Vitamin D on falls: a meta-analysis. JAMA 2004, 291(16):1999-2006.

33. Boonen S, Bischoff-Ferrari HA, Cooper C, Lips P, Ljunggren O, Meunier PJ, Reginster JY: Addressing the musculoskeletal components of fracture risk with calcium and vitamin D: a review of the evidence. Calcif Tissue Int 2006, 78(5):257-270

34. Broe KE, Chen TC, Weinberg J, Bischoff-Ferrari HA, Holick MF, Kiel DP: A higher dose of vitamin d reduces the risk of falls in nursing home residents: a randomized, multiple-dose study. J Am Geriatr Soc 2007, 55(2):234-239.

35. Gennari C: Calcium and vitamin D nutrition and bone disease of the elderly. Public Health Nutr 2001, 4(2B):547-559.

36. Lips P, Bouillon R, van Schoor NM, Vanderschueren D, Verschueren S, Kuchuk N, Milisen K, Boonen S: Reducing fracture risk with calcium and vitamin D. Clin Endocrinol (Oxf) 2010, 73(3):277-285.

37. Malabanan AO, Holick MF: Vitamin D and bone health in postmenopausal women. J Womens Health (Larchmt) 2003, 12(2):151-156.

38. Rodriguez-Martinez MA, Garcia-Cohen EC: Role of $\mathrm{Ca}(2+)$ and vitamin D in the prevention and treatment of osteoporosis. Pharmacol Ther 2002, 93(1):37-49.

39. Edwards MH, Jameson K, Denison H, Harvey NC, Sayer AA, Dennison EM Cooper C: Clinical risk factors, bone density and fall history in the prediction of incident fracture among men and women. Bone 2013, 52(2):541-547

40. Pryor T, Wiederman MW, McGilley B: Laxative abuse among women with eating disorders: an indication of psychopathology? Int J Eat Disord 1996, 20(1):13-18.

41. Vanin JR, Saylor KE: Laxative abuse: a hazardous habit for weight control. J Am Coll Health 1989, 37(5):227-230.

42. Campbell AJ, Busby WJ, Horwath CC: Factors associated with constipation in a community based sample of people aged 70 years and over. J Epidemiol Community Health 1993, 47(1):23-26.

43. Everhart JE, Go VL, Johannes RS, Fitzsimmons SC, Roth HP, White LR: A longitudinal survey of self-reported bowel habits in the United States. Dig Dis Sci 1989, 34(8):1153-1162.

44. Koch T, Hudson S: Older people and laxative use: literature review and pilot study report. J Clin Nurs 2000, 9(4):516-525.

45. McCrea GL, Miaskowski C, Stotts NA, Macera L, Paul SM, Varma MG: Gender differences in self-reported constipation characteristics, symptoms, and bowel and dietary habits among patients attending a specialty clinic for constipation. Gend Med 2009, 6(1):259-271.

46. Design of the Women's Health Initiative clinical trial and observational study: The Women's Health Initiative Study Group. Control Clin Trials 1998, 19(1):61-109.

47. Jackson RD, LaCroix AZ, Gass M, Wallace RB, Robbins J, Lewis CE, Bassford T, Beresford SA, Black HR, Blanchette $P$, et al: Calcium plus vitamin $\mathrm{D}$ supplementation and the risk of fractures. $N$ Engl J Med 2006, 354(7):669-683.

48. Langer RD, White E, Lewis CE, Kotchen JM, Hendrix SL, Trevisan M: The Women's Health Initiative Observational Study: baseline characteristics of participants and reliability of baseline measures. Ann Epidemio/ 2003, 13(9 Suppl):S107-121.

49. Prentice RL, Caan B, Chlebowski RT, Patterson R, Kuller LH, Ockene JK, Margolis KL, Limacher MC, Manson JE, Parker LM, et al: Low-fat dietary pattern and risk of invasive breast cancer: the Women's Health Initiative Randomized Controlled Dietary Modification Trial. JAMA 2006, 295(6):629-642.
50. Rossouw JE, Anderson GL, Prentice RL, LaCroix AZ, Kooperberg C, Stefanick ML, Jackson RD, Beresford SA, Howard BV, Johnson KC, et al: Risks and benefits of estrogen plus progestin in healthy postmenopausal women: principal results From the Women's Health Initiative randomized controlled trial. JAMA 2002, 288(3):321-333.

51. Hays J, Hunt JR, Hubbell FA, Anderson GL, Limacher M, Allen C, Rossouw JE: The Women's Health Initiative recruitment methods and results. Ann Epidemiol 2003, 13(9 Suppl):S18-77.

52. Masud T, Morris RO: Epidemiology of falls. Age Ageing 2001 30(Suppl 4):3-7.

53. Stalenhoef PA, Diederiks JP, Knottnerus JA, Kester AD, Crebolder HF: A risk model for the prediction of recurrent falls in community-dwelling elderly: a prospective cohort study. J Clin Epidemiol 2002, 55(11):1088-1094.

54. Ainsworth BE, Haskell WL, Leon AS, Jacobs DR Jr, Montoye HJ, Sallis JF, Paffenbarger RS Jr: Compendium of physical activities: classification of energy costs of human physical activities. Med Sci Sports Exerc 1993, 25(1):71-80.

55. Kristal AR, Feng Z, Coates RJ, Oberman A, George V: Associations of race/ ethnicity, education, and dietary intervention with the validity and reliability of a food frequency questionnaire: the Women's Health Trial Feasibility Study in Minority Populations. Am J Epidemiol 1997, 146(10):856-869.

56. Ware JE Jr, Sherbourne CD: The MOS 36-item short-form health survey (SF-36). I. Conceptual framework and item selection. Med Care 1992, 30(6):473-483

57. Gray SL, LaCroix AZ, Larson J, Robbins J, Cauley JA, Manson JE, Chen Z Proton pump inhibitor use, hip fracture, and change in bone mineral density in postmenopausal women: results from the Women's Health Initiative. Arch Intern Med 2010, 170(9):765-771.

58. LaCroix AZ, Cauley JA, Pettinger M, Hsia J, Bauer DC, McGowan J, Chen Z, Lewis CE, McNeeley SG, Passaro MD, et al: Statin use, clinical fracture, and bone density in postmenopausal women: results from the Women's Health Initiative Observational Study. Ann Intern Med 2003, 139(2):97-104

59. Cumming RG, Miller JP, Kelsey JL, Davis P, Arfken CL, Birge SJ, Peck WA: Medications and multiple falls in elderly people: the St Louis OASIS study. Age Ageing 1991, 20(6):455-461.

60. Lavsa SM, Fabian TJ, Saul MI, Corman SL, Coley KC: Influence of medications and diagnoses on fall risk in psychiatric inpatients. Am J Health Syst Pharm 2010, 67(15):1274-1280.

61. Sandhu HS, Gilles E, DeVita MV, Panagopoulos G, Michelis MF: Hyponatremia associated with large-bone fracture in elderly patients. Int Urol Nephrol 2009, 41(3):733-737.

62. Gross P: Treatment of hyponatremia. Intern Med 2008, 47(10):885-891.

63. Renneboog B, Musch W, Vandemergel X, Manto MU, Decaux G: Mild chronic hyponatremia is associated with falls, unsteadiness, and attention deficits. Am J Med 2006, 119(1):71 e71-78.

64. Shumway-Cook A, Ciol MA, Hoffman J, Dudgeon BJ, Yorkston K, Chan L: Falls in the Medicare population: incidence, associated factors, and impact on health care. Phys Ther 2009, 89(4):324-332.

65. Robbins J, Aragaki AK, Kooperberg C, Watts N, Wactawski-Wende J, Jackson $R D$, LeBoff MS, Lewis CE, Chen Z, Stefanick ML, et al: Factors associated with 5-year risk of hip fracture in postmenopausal women. JAMA 2007, 298(20):2389-2398.

66. Bea JW, Zhao Q, Cauley JA, LaCroix AZ, Bassford T, Lewis CE, Jackson RD, Tylavsky FA, Chen Z: Effect of hormone therapy on lean body mass, falls, and fractures: 6-year results from the Women's Health Initiative hormone trials. Menopause 2011, 18(1):44-52.

doi:10.1186/1471-2318-13-38

Cite this article as: Haring et al.: Laxative use and incident falls, fractures and change in bone mineral density in postmenopausal women: results from the Women's Health Initiative. BMC Geriatrics 2013 13:38 